“C 2017 IEEE. Personal use of this material is permitted. Permission from IEEE must be obtained for all other uses, in any current or future media, including reprinting/republishing this material for advertising or promotional purposes, creating new collective works, for resale or redistribution to servers or lists, or reuse of any copyrighted component of this work in other works." 


\title{
Modeling and Measurement of Magnetic Hysteresis of Soft Magnetic Composite Materials under Different Magnetizations
}

\author{
Weijie Xu, Student Member, IEEE, Nana Duan, Student Member, IEEE, Shuhong Wang, Senior \\ Member, IEEE, Youguang Guo, Senior Member, and Jianguo Zhu, Senior Member, IEEE
}

\begin{abstract}
Soft magnetic composite (SMC) materials and their application in electromagnetic devices have undergone significant development due to their unique advantages such as low eddy current loss, quasi-isotropy of mechanical and magnetic properties, low cost and low material consumption during the production process. However, the magnetic properties of SMC materials are very different from that of the laminated SiFe materials, which are particularly challenging for the design and application of electrical machines. This paper presents the modeling and measurement of magnetic properties of SMC materials under both alternating and rotational magnetic excitations. Based on the underlying magnetization mechanisms, a vectorial elemental operator with biaxial anisotropy is introduced, and the concept of distribution function is utilized to describe the density of operators in the specimen. To verify this proposed model, the magnetic hysteresis of SMC material is simulated and compared with the experimental results obtained by the 3-D magnetic property measurement system. The good agreement shows the validity and practicability of this vectorial elemental operator.
\end{abstract}

Index Terms-Elemental operator, magnetic properties, soft magnetic composite (SMC) materials, vectorial hysteresis model.

\section{INTRODUCTION}

$\mathrm{T}$ HE soft magnetic composite (SMC) materials, produced by powder metallurgical techniques, are composed of iron powders (about $100 \mu \mathrm{m}$ in diameter) of high purity and surface coating for electrical insulation and mechanical bonding [1]. The coated iron powders, with high electrical resistivity, are pressed into a solid magnetic core after a series of technical

Manuscript received December 31, 2015; revised April 11, 2016, and May 8, 2016; accepted May 18, 2016. This work was supported in part by the China Scholarship Council (No: 201306280073).

W. J. Xu, N. N. Duan, and S. H. Wang are with the School of Electrical Engineering, Xi'an Jiaotong University, Xi'an 710049, China (e-mail: xuweijie@outlook.com; duannana@outlook.com; shwang@mail.xjtu.edu.cn).

Y. G. Guo, and J. G. Zhu are with the Faculty of Electrical, Mechanical and Mechatronic Systems, University of Technology Sydney, Sydney, NSW 2007, Australia (e-mail: Youguang.Guo-1@uts.edu.au; Jianguo.Zhu@uts.edu.au). steps, such as the die pressing, heating and curing treatment [2]. Thus, compared with the laminated silicon-steel sheets, the SMC materials have many unique characteristics and advantages such as low eddy current loss, quasi-isotropy of mechanical, magnetic and thermal properties, great design flexibility, low cost and low material consumption during the production process [3].

In the laminated steel sheets, the magnetic flux can be designed to flow only within the lamination plane for its thin structure [4], [5]. If the magnetic flux is perpendicular to the laminations, great eddy-current loss would be produced during the magnetization process [6]-[8]. Hence, the laminated steel sheets are not suitable for some special electromagnetic devices with 3-D magnetic flux paths, such as transverse flux, claw pole, and axial flux permanent magnet motors [9]-[11]. To lift these restrictions, the SMC materials can be employed for fabricating electromagnetic devices of special structures [12]. Because of the powdered nature, the magnetic property of SMC material is naturally quasi-isotropic, although slight magnetic anisotropic property would be generated due to the pressing and heat treatment in the preparation process of SMC cores [13].

Since the SMC materials are being used in more and more applications with satisfaction, the magnetic properties, such as the relationship between the magnetic field strength $(\mathbf{H})$ and the magnetic flux density (B) or magnetization (M) and the specific core loss under the one-dimensional (alternating field) and/or two-dimensional (rotational field) magnetizations, need to be fully understood for developing high performance electromagnetic devices.

Furthermore, the core loss (mainly hysteresis loss) in the SMC electrical machine is comparable with the copper loss [14]. This is vastly different from the traditional laminated steel machines, in which the copper loss takes up a high proportion of nearly $90 \%$ of the total power loss [15]-[17]. Hence, accurate computation and simulation of magnetic hysteresis in SMC materials are crucial, and should be properly taken into account in the design of electromagnetic devices.

To complicate the matter, due to the complex mechanism of the magnetic hysteresis, the $\mathbf{B}$ and $\mathbf{H}$ do not always align in the same direction during the process of magnetization, especially under the rotational excitation field [18]. Various hysteresis models have been proposed, such as the Stoner-Wohlfarth (S-W) model [19], the Preisach model [20], and the Della Torre-Pinzaglia-Cardelli (DPC) model [21]. Among them, the 
Preisach model is a scalar hysteresis model, and thus difficult to describe the vectorial relationship between $\mathbf{H}$ and B [22]. The $\mathrm{S}-\mathrm{W}$ model based on the $\mathrm{S}-\mathrm{W}$ particle with uniaxial anisotropy is inconsistent with the physical mechanisms of SMC materials [23]. The phenomenological vector hysteresis operator (hysteron) in DPC model has weak appeal to physical intuition [24]. Therefore, the vectorial magnetic properties of SMC material have not been successfully described and analyzed, not to mention the application for engineering practice.

Based on the magnetization mechanisms of the SMC materials, a vectorial elemental operator (the core of the novel hysteresis model) is derived. The analytical expressions of the vectorial relationship between $\mathbf{H}$ and $\mathbf{M}$ of a single elemental operator under alternating and rotational fluxes are then deduced, respectively. The interaction field and the coercive force of each elemental operator can be considered in terms of the distribution function of elemental operators. The magnetic properties under different vectorial magnetizations can be finally obtained by integrating the effects of elemental operators according to the distribution. To verify the proposed model, the magnetic properties of SOMALOY ${ }^{\mathrm{TM}} 500$, a classical type of SMC material, are simulated and compared with the experimental results.

\section{Modelling of Magnetic Properties of SMC MATERIAL}

\section{A. The Vectorial Elemental Operator}

In this model, it is assumed that each surface coating iron powder in SMC material can be regarded as a microscopic crystalline particle with magnetocrystalline anisotropy, i.e. multiple hard and easy axes. Inside each particle, there exist various magnetic domains, and each domain consists of a great number of dipoles with magnetization $\mathbf{m}$. Fig.1 shows the magnetically hard, medium and easy axes of a unit cell of body-centered cubic (bcc) iron [25]. In this paper, only the two dimensional magnetization process in the (100) plane is investigated.

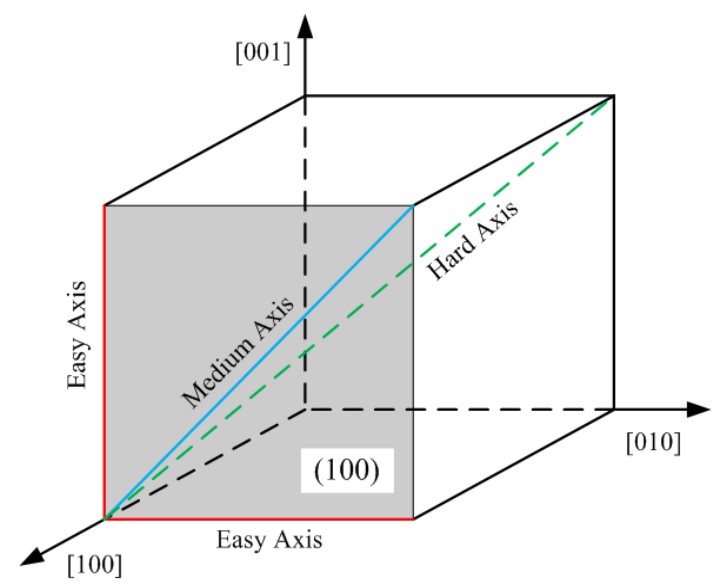

Fig. 1. Different magnetization axes of an iron particle with cubic anisotropy.

Due to the spin-lattice coupling in cubic crystals, the anisotropy energy density has cubic symmetry, as shown in Fig.
2. When the magnetic field $\mathbf{H}$ is rotating in the crystal with a fixed magnitude, both the magnitude and direction of the magnetization, $\mathbf{m}$, may vary. If the magnetization is confined to the (100) plane which has biaxial anisotropy, the total energy on this plane can be expressed as

$$
E=K \sin ^{2} \theta \cos ^{2} \theta
$$

where $\theta$ is the angle of magnetization relative to the easy axis, and $K$ is the biaxial anisotropy coefficient of the (100) plane.

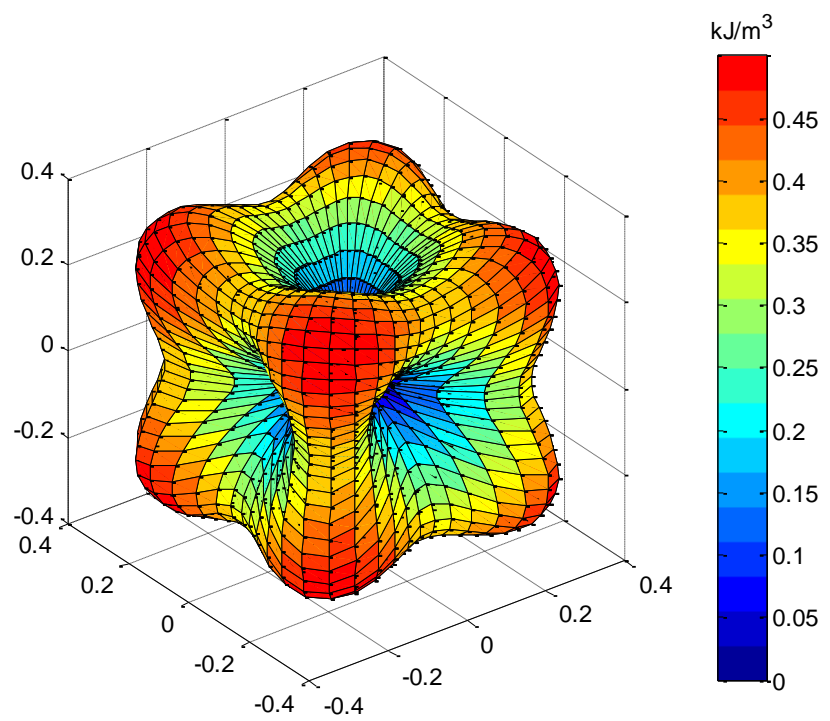

Fig. 2. Cubic anisotropy energy density of a cubic textured magnetic material (anisotropy coefficient $K>0$ ).

Based on the assumption that SMC materials are composed of a large amount of interacting magnetic dipoles which possess different biaxial anisotropy coefficients, the magnetization orientation of each dipole can be determined from the energy contributions of the applied field and anisotropy [26]. The magnetic dipole in the particle, which has the biaxial anisotropy, can be defined as a vectorial elemental operator. Thus, the total energy of this elemental operator can be written as

$$
E=K \sin ^{2} \theta \cos ^{2} \theta-\mu_{0} \mathrm{Hm} \cos \left(\theta_{H}-\theta\right)
$$

where $\theta$ and $\theta_{H}$ are the angles of the resultant magnetization and the applied field, respectively, both with respect to the operator's easy axis, and $\mathbf{m}$ is the magnetization of the operator, which can be deducted from the anisotropy energy. The first term is the biaxial anisotropic energy of the elemental operator, and the second the interaction energy associated with the applied magnetic field and the elemental operator's magnetization.

Different from the S-W particle with uniaxial anisotropy, the biaxial anisotropy is adopted in this vectorial elemental operator to be consistent with the magnetization mechanism of the SMC material. However, the stable orientation of the vectorial magnetization $\mathbf{m}$ can also be determined by minimizing the total 
energy of the elemental operator as

$$
\begin{aligned}
& \frac{\partial E}{\partial \theta}=\frac{1}{2} K \sin 4 \theta-\mu_{0} \mathrm{Hm} \sin \left(\theta_{H}-\theta\right)=0 \\
& \frac{\partial^{2} E}{\partial \theta^{2}}=2 K \cos 4 \theta+\mu_{0} \mathrm{Hm} \cos \left(\theta_{H}-\theta\right)=0
\end{aligned}
$$

Solving the above equations, one obtains the expression of a biasteroid curve (also called wind rose) as the following

$$
\begin{aligned}
& \mathrm{H}_{x}=\frac{2 K}{\mu_{0} \mathrm{~m}} \cos ^{3} \theta\left(5-6 \cos ^{2} \theta\right) \\
& \mathrm{H}_{y}=\frac{2 K}{\mu_{0} \mathrm{~m}} \sin ^{3} \theta\left(5-6 \sin ^{2} \theta\right)
\end{aligned}
$$

(6)

where $\mathrm{H}_{x}$ and $\mathrm{H}_{y}$ are the components of the applied field $\mathbf{H}$ along the two orthogonal easy axes, respectively.

The biasteroid curve, as shown in Fig. 3, has biaxial anisotropy with two orthogonal hard axes and two orthogonal easy axes. This characteristic is strictly consistent with the physical mechanism of the SMC materials which have biaxial anisotropy on each crystal plane.

Similar to the asteroid of the S-W model, graphical interpretation can be given for describing the magnetization process of this biaxial elemental operator. The biasteroid curve separates the $\mathrm{H}_{\mathrm{x}}-\mathrm{H}_{\mathrm{y}}$ plane into four regions in accordance to the number of energy minima, as shown in Fig. 4.

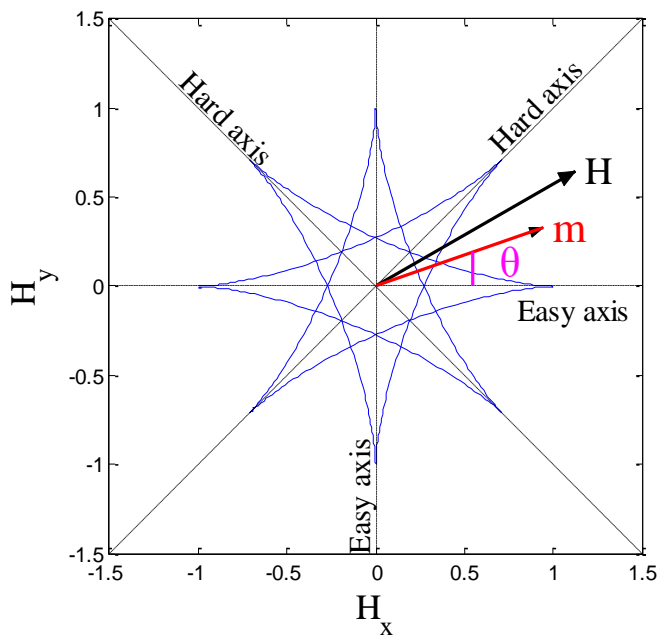

Fig. 3. The biasteroid shape of the biaxial elemental operator with two orthogonal easy axes and two orthogonal hard axes.

Figs. 4(a)-(c) show the situations when the magnitude of applied magnetic field is inside the biasteroid shape. As the applied field $\mathbf{H}$ increases, there exist four, three and two energy minima. The establishment of the only one actual energy minimum is closely related to the magnetization history. In Figs. 4(d) and (e), when the magnitude of applied magnetic field $\mathbf{H}$ is outside the biasteroid curve, the magnetization has only one stable orientation as there is only one energy minimum, and the magnetization $\mathbf{m}$ moves closer to the magnetic field $\mathbf{H}$.

\section{B. Vectorial Elemental Operator under Alternating Field}

To clearly describe the vectorial magnetic properties of the elemental operator under alternating applied field, the vectorial magnetization $\mathbf{m}$ of the elemental operator can be divided into two components, the transverse and longitudinal magnetization components, which are the projections of $\mathbf{m}$ perpendicularly to and along with $\mathbf{H}$, respectively.
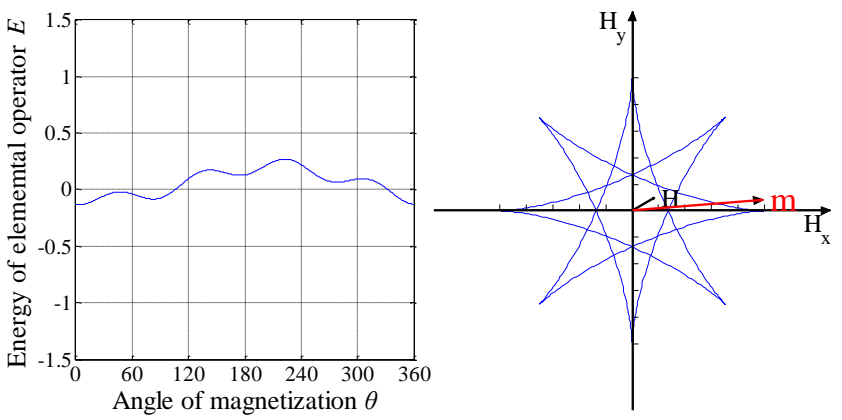

(a)
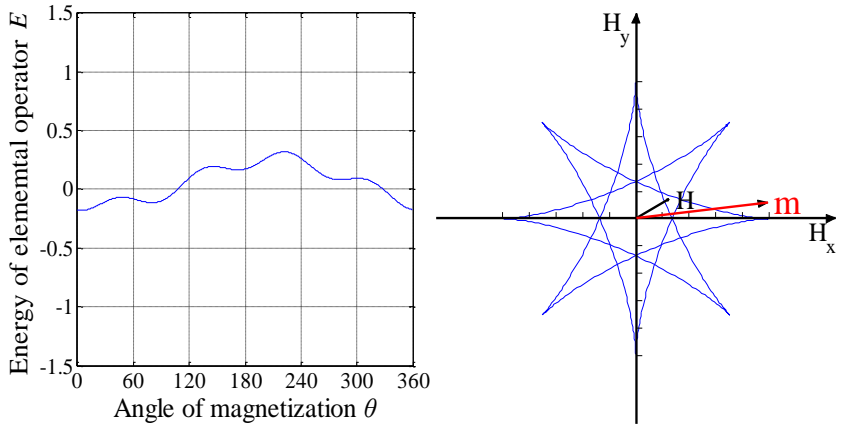

(b)

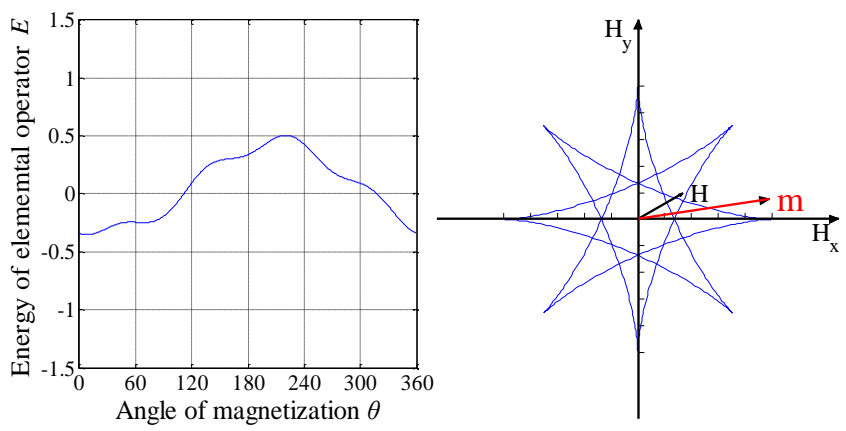




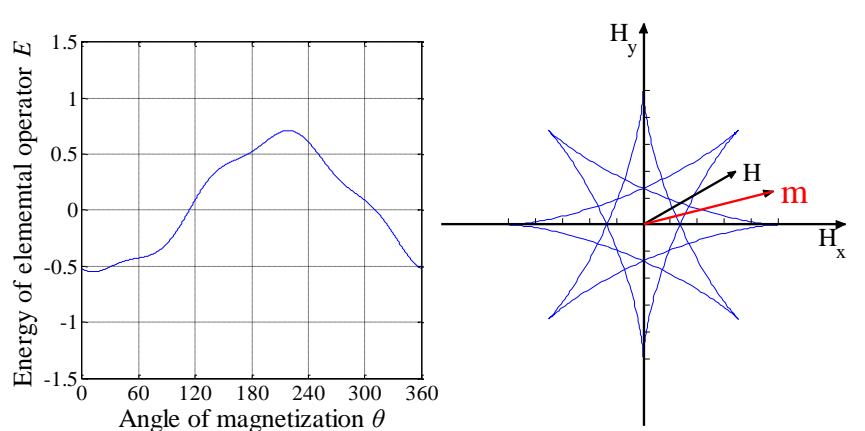

(d)

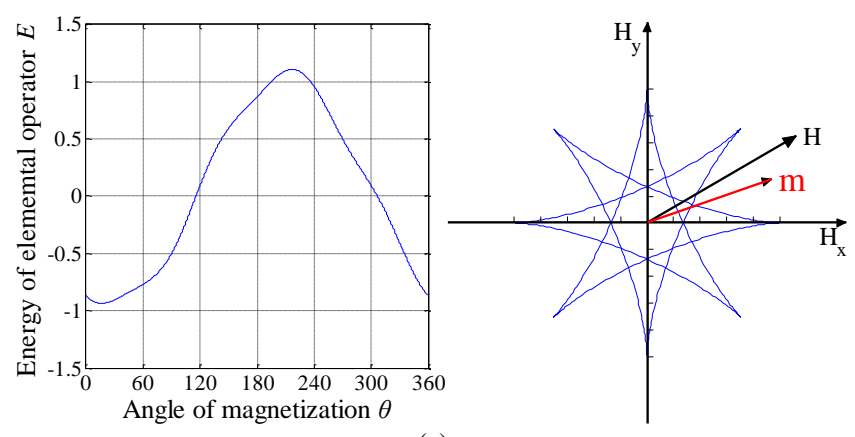

(e)

Fig. 4. Energy density and the magnetization behavior of the biaxial elemental operator with the applied field increasing along the fixed angle $\theta=30^{\circ}$. The number of local energy minima which determined by the vectorial applied field is (a) four, (b) three, (c) two, and one in (d) and (e).

The longitudinal and transverse magnetization components for different orientations of the applied field are illustrated in Figs. 5 and 6, respectively. For convenience, the magnetic field and magnetization can be normalized by its saturation value, such that $\mathbf{h}=\mathbf{H} / \mathrm{H}_{\mathrm{sat}}$. Note that the orientation of external field relative to the easy axis must not exceed the $45^{\circ}$ line, since the elemental operator has two orthogonal hard axes and two orthogonal easy axes, and the largest angle between the easy axis and the hard axis is $45^{\circ}$.

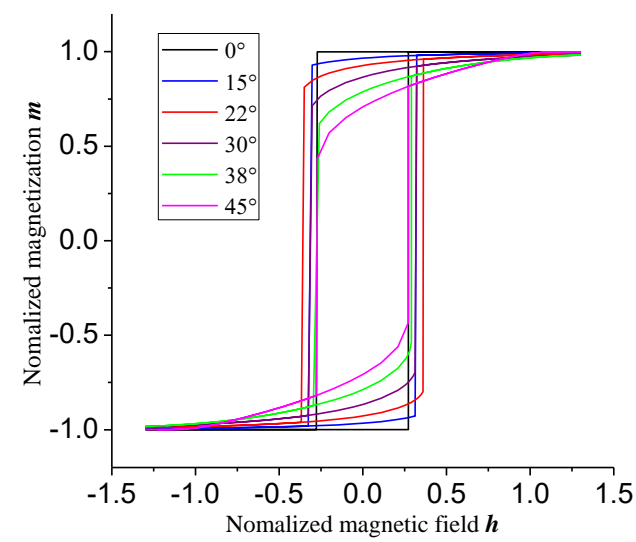

Fig. 5. The longitudinal hysteresis loops of vectorial elemental operator for different applied magnetic field orientation.

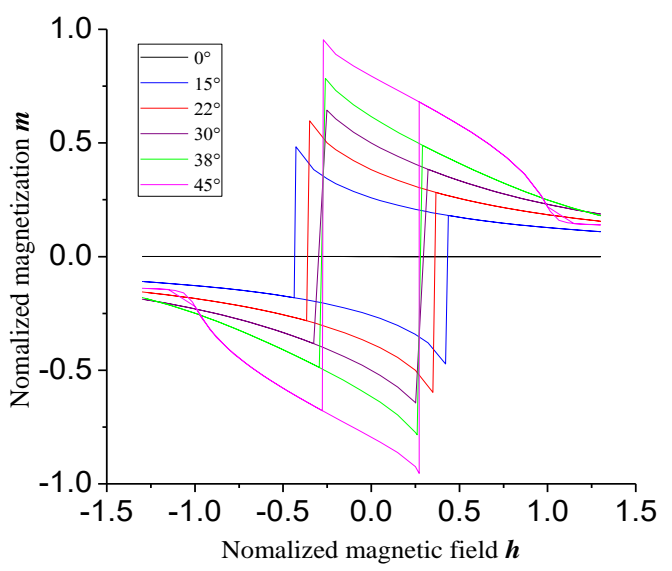

Fig. 6. The transverse hysteresis loops of vectorial elemental operator for different applied magnetic field orientation.

Similar to the S-W model, a square longitudinal hysteresis loop can be obtained when the external field $\mathbf{H}$ is parallel to the easy axis, as shown in Fig. 5, which is exactly the conventional Preisach rectangular hysteron. Thus, this vectorial elemental operator can also be adopted to model the scalar magnetic hysteresis by restricting the applied magnetic field to vary along one dimension.

To keep the consistency of the potential physical mechanism in the magnetization process, the interaction field $\mathrm{h}_{i}$, which is the synthetic field from neighboring elemental operators, must be taken into account. As shown in Fig. 7, the shift of biasteroid curve depends greatly on the interaction field, and the corresponding hysteresis loop is similar to the Preisach hysteon under interaction field.

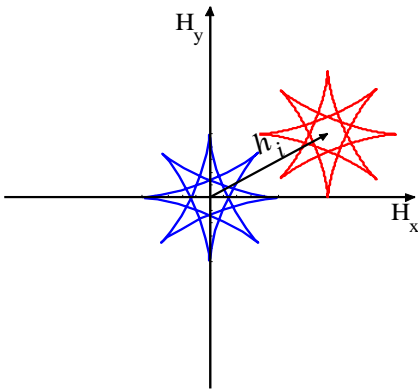

(b)

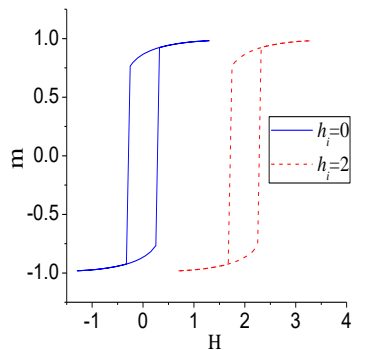

(a)
Fig. 7. (a) The positional change of the elemental operator and (b) the corresponding longitudinal hysteresis loop vary with the interaction field.

Another indispensable factor affecting the performance of the elemental operator is the crystal anisotropy. As shown in Fig. 8, the shape of biasteroid curve varies with the anisotropy constant, and so does the coercivity of the corresponding hysteresis loop.

An analytical expression of the alternating field $\mathbf{H}$ and the magnetization $\mathbf{m}$ has been presented in [27]. However, this expression can only describe the uniaxial anisotropy S-W operator, and it ignores the magnetic interactions between the elemental operators with biaxial crystal anisotropy. Thus, in the frame of partial approximate substitutions, a modified analytical expression of the applied alternating field $\mathbf{H}$ and the magnetization $\mathbf{m}$ of an elemental operator can be presented as 


$$
m\left(H, \theta_{H}\right)= \pm \frac{\frac{\left(H+h_{i}\right)}{h_{k}} \pm \cos \theta_{H}}{\sqrt{\frac{\left(H+h_{i}\right)^{2}}{h_{k}} \pm 2 \frac{\left(H+h_{i}\right)}{h_{k}} \cos \theta_{H}+1}}
$$

where $h_{k}=\frac{2 K}{\mu_{0} \mathrm{M}_{s}}$ is the normalized anisotropy field which corresponding to the coercivity for each elemental operator, and $h_{i}$ the interaction field on the elemental operator. The plus sign is for the upward branch and the minus sign for the downward branch in a given hysteresis loop.

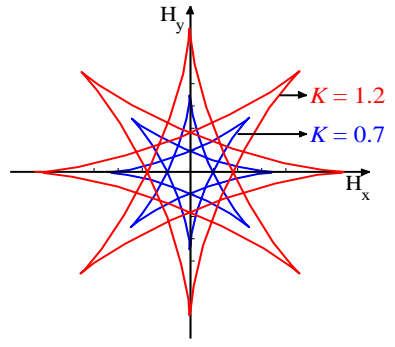

(a)

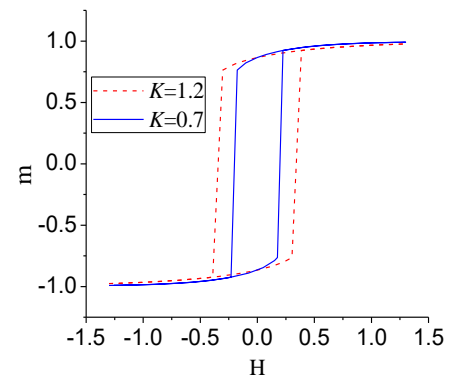

(b)
Fig. 8. (a) The shape of biasteroid and (b) its corresponding longitudinal hysteresis loop vary with the anisotropy coefficient.

Assuming that the SMC material is composed by lots of interacting elemental operators with different biaxial crystal anisotropy, the bulk magnetization $\mathbf{M}$ for a given applied alternating field $\mathbf{H}$ can be obtained by the following integral:

$$
M(H)=\iiint m(H, \theta) P\left(h_{i}, h_{k}\right) \cos \theta \mathrm{d} h_{i} \mathrm{~d} h_{k} \mathrm{~d} \theta
$$

where $P\left(h_{i}, h_{k}\right)$ is the distribution function of the elemental operators in the SMC material.

To simulate the magnetic properities of SMC material SOMALOY $^{\mathrm{TM}} 500$, the distribution can be described by a 2-D Gaussian distribution which is assumed to be a product of two Gaussian distributions that are independent to each other, as shown in Fig. 9. The expression of this function is defined as follows:

$$
P\left(h_{i}, h_{k}\right)=N \frac{1}{2 \pi \sigma_{i} \sigma_{k}} \exp \left(-\frac{h_{i}^{2}}{2 \sigma_{i}^{2}}-\frac{\left(h_{k}-\mu_{k}\right)^{2}}{2 \sigma_{k}^{2}}\right)
$$

where $N$ is the normalization factor, $\mu_{k}$ the center of distribution for the anisotropy field, $\sigma_{k}$ and $\sigma_{i}$ are the standard deviation of the normalized anisotropy field $h_{k}$ and the interaction field $h_{i}$, respectively.

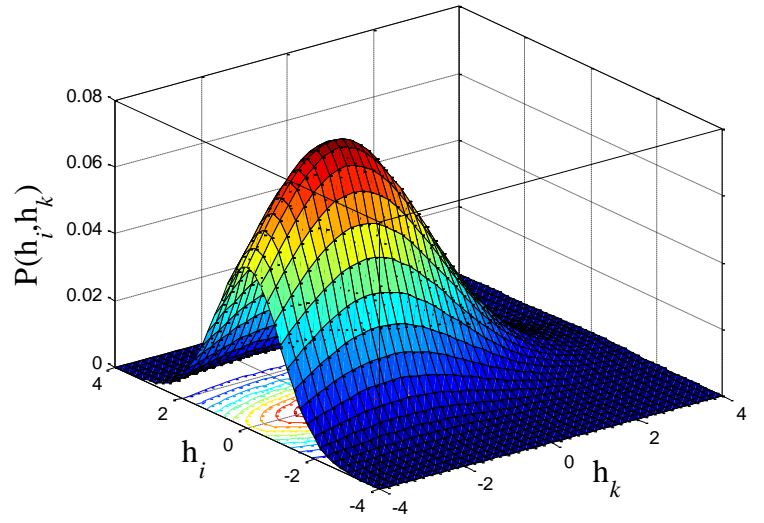

Fig. 9. The 2-D Gaussian distribution function of the elemental operators under alternating field.

In this study, all the parameters of this 2-D Gaussian distribution function can be determined numerically by fitting the model to the limiting hysteresis loop. With the comparison of the key points (saturation, residual magnetism, and coercive force) on the measured and simulated limiting hysteresis loop, the parameters of distribution function can be chosen while the error is suitably small.

\section{Vectorial Elemental Operator under Rotational Field}

In some specific part of the electromagnetic devices, such as the cores of rotating electrical AC machines and the T-joints of multi-phase transformers, the magnetic flux rotates. The design and analysis of such devices require the modelling and experimental measurement of vectorial magnetic properties, which are totally different with the case under alternating field. In this part, these phenomena are explained and modeled based on the vectorial elemental operator.

When a rotating magnetic field $\mathbf{H}$, with fixed magnitude but varying rotation angle, is applied on an elemental operator, the corresponding magnetization will follow the rotation but with different rotation angle and generate varying magnitude, as illustrated in Fig. 10. Thus, the flower-shaped loci of the magnetization $\mathbf{m}$ will be obtained under the normalized rotational magnetic field $\mathbf{h}$.

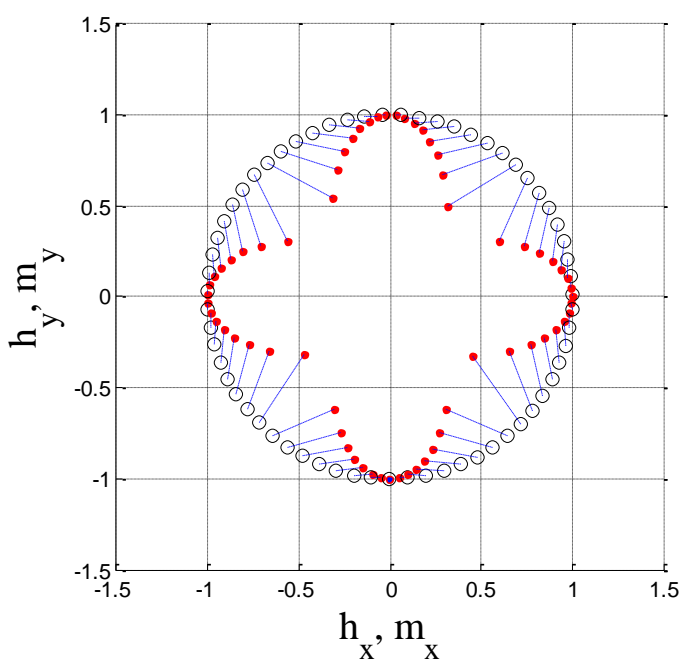


Fig. 10. The performance of an elemental operator in one period of rotational applied magnetic field: the blue dashed lines connect the normalized applied magnetic field (black circles) and the corresponding magnetization (red dots).

In another case, if we control the elemental operator to work under the circularly rotational flux $\mathbf{B}$, in other words, make the generated magnetization $\mathbf{M}$ have a fixed magnitude, the magnetic field $\mathbf{H}$ will also follow the rotation and produce a flower-shaped trajectory, as illustrated in Fig. 11.

The flower-shaped trajectory is directly related to the anisotropy of the elemental operator. Different anisotropy coefficients correspond to different shapes of the trajectory. As shown in Fig. 12, the smaller the anisotropy coefficient, the closer the shape of the loci to the circular shape.

It is assumed that under the rotating field, the interaction from other elemental operators will deflect the elemental operator Meanwhile, an inclined angle will occur on the loci of the applied field as shown in Fig. 13.

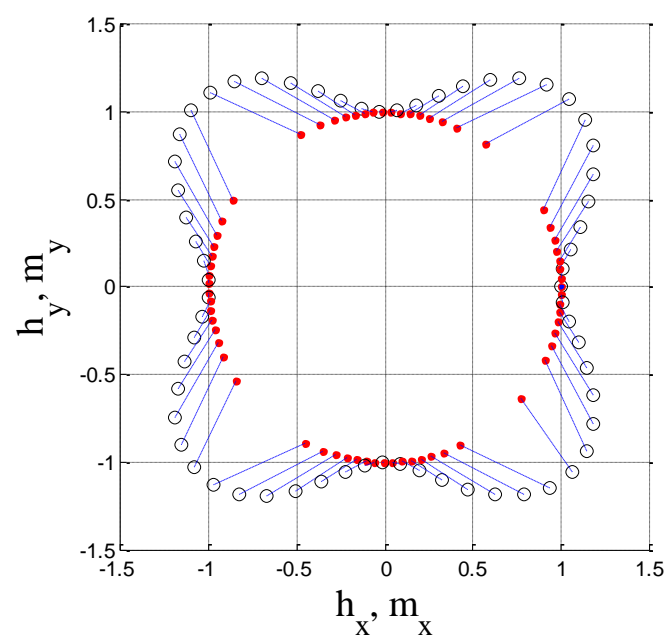

Fig. 11. The performance of an elemental operator in one period of circularly rotational magnetic flux: the blue dashed lines connect the normalized magnetization (red dots) and the corresponding applied magnetic field (black circles).

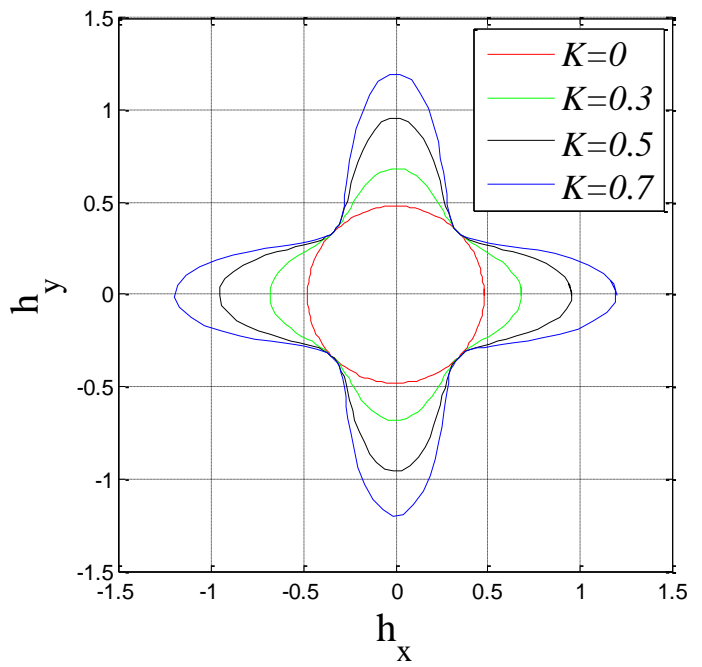

Fig. 12. The flower-shaped trajectories of the applied field correspond to different anisotropy coefficients. When $K=0$, the shape is circular which means the 2-D isotropy of the elemental operator.

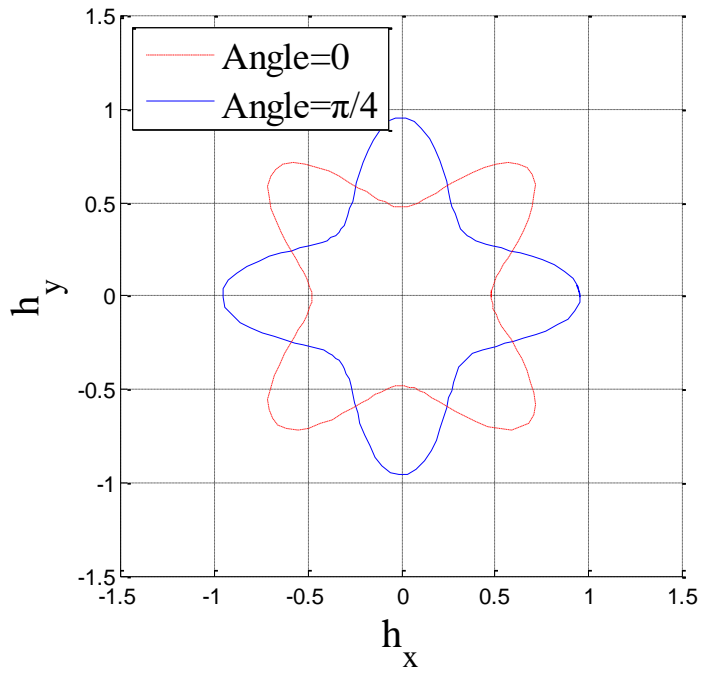

Fig. 13. An incline occurs on the loci of the applied field after taking into account the interaction.

To describe the vectorial relationship between $\mathbf{H}$ and $\mathbf{m}$ of a single elemental operator under rotational flux and the flower-shape of the loci, an analytical expression is proposed as the following

$$
m(\theta, K, \alpha)=M_{S}\left[k \sin ^{2} 2(\theta+\alpha)+\cos ^{2} 2(\theta+\alpha)\right]\left|\frac{h_{\mathrm{m}}}{N_{k}}\right|
$$

where $k$ is the coefficient related to the anisotropy of the elemental operator, $M_{\mathrm{s}}$ the saturation magnetization along the easy axis, $\alpha$ the inclination angle of elemental operator, $h_{\mathrm{m}}=\mathrm{H}$ $\cos \left(\theta_{H^{-}} \theta\right) / \mathrm{H}_{\text {sat }}$ the normalized projection of the magnetic field $\mathbf{H}$ on the direction of magnetization $\mathbf{M}, N_{\mathrm{k}}=0.45 h^{2}-0.56 h+$ 0.35 a correction factor in this expression, and $h$ the normalized magnetic field.

Meanwhile, the orientation of the magnetization $\theta$ and the angle $\theta_{H^{-}} \theta$, which is the angle between magnetic field $\mathbf{H}$ and magnetization $\mathbf{M}$, can be determined by the biasteroid curve method, as illustrated in Fig. 3. Therefore, in this rotational magnetization, both the directions and magnitudes of the $\mathbf{H}$ and $\mathbf{M}$ may vary, as well as the directional angle difference between $\mathbf{H}$ and $\mathbf{M}$, which means $\mathbf{H}$ may either be ahead or lag behind of $\mathbf{M}$. This vectorial relationship between $\mathbf{H}$ and $\mathbf{M}$ can be defined as the rotational hysteresis. In addition, this vectorial property can produce the hysteresis loss due to the rotational magnetization.

Similar to the scalar case, the 2-D Gaussian distribution can be adopted to describe the distribution of the anisotropy and the inclination angle of the elemental operator in the rotating magnetic field. The vectorial magnetization $\mathbf{M}$ for a given rotational field $\mathbf{H}$ at an arbitrary angle $\theta$ can be calculated by the superposition of elemental operators, as shown in (8) above.

\section{Measurement of Magnetic Properties of SMC MATERIAL}

The magnetic properties of SMC material under alternating field (1-D) and rotational field (2-D) can be measured by using the 3-D magnetic property measurement system [28], [29]. 
Fig. 14 illustrates the block diagram of measurement system, which consists of a 3-D magnetic property tester, a 3 channel power amplifier, and a data acquisition and control unit.

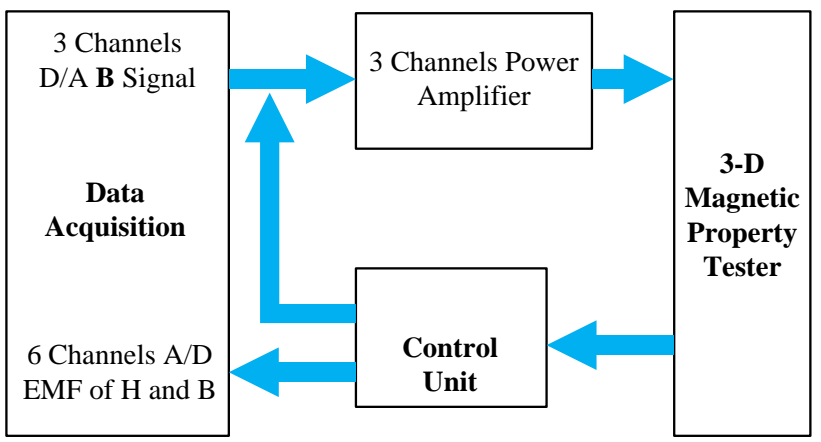

Fig. 14. The functional block diagram of 3-D magnetic property measurement system.

Fig. 15 depicts the schematic diagram of the structure and a photo of the 3-D tester [30]. By controlling the magnetic excitation in three axes, this tester can produce different magnetic flux patterns, e.g. alternating flux in arbitrary direction, rotational flux in arbitrary plane or 3-D space, according to the requirement of the measurement.

In order to measure the magnetic properties of SMC material and verify the proposed model, SOMALOY ${ }^{\mathrm{TM}} 500$, a kind of SMC material, is measured under different magnetic flux patterns by this 3 -D magnetic property measurement system [31].

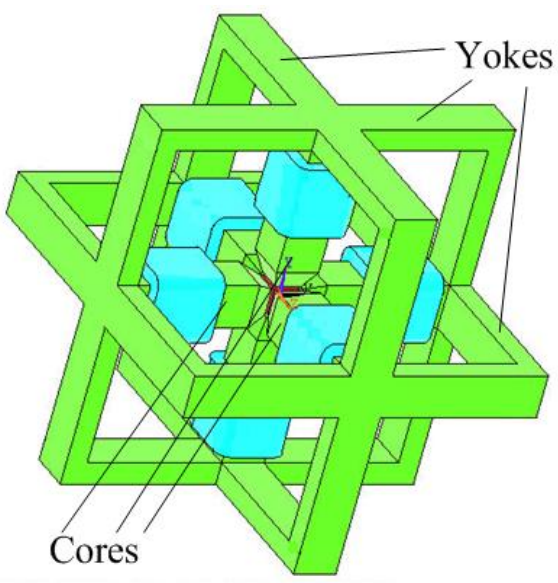

(a)

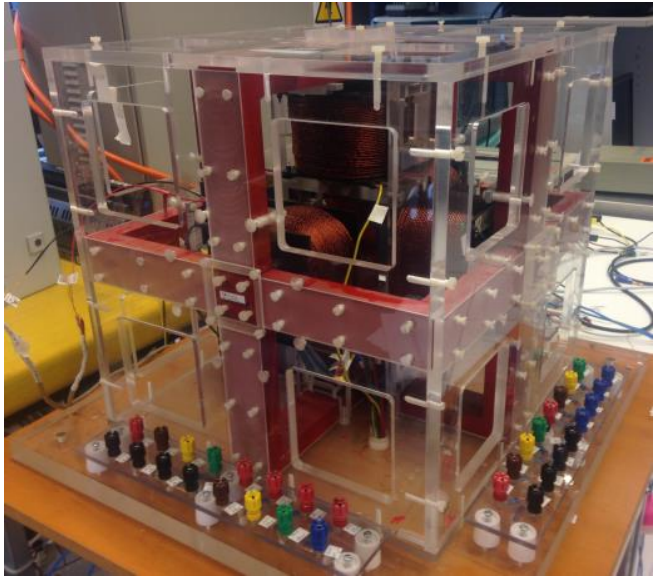

(b)

Fig. 15. (a) The schematic diagram, and (b) the real structure of the 3-D magnetic property tester.

For SOMALOY ${ }^{\mathrm{TM}} 500$, the parameters of the 2-D Gaussian distribution function in the proposed model are $\sigma_{i}=0.04, \sigma_{k}=$ 0.43 and $\mu_{k}=0.011$, which can be directly determined by fitting the measured limiting hysteresis loop of the material.

Fig. 16(a) depicts the comparison between the simulated and measured limiting hysteresis loop of SOMALOY ${ }^{\mathrm{TM}}$ 500. Fig. 16(b) plots the comparison between the simulated and measured major hysteresis loops with different flux densities at $50 \mathrm{~Hz}$. For better readability and the symmetry of the hysteresis loop, only the upper-half hysteresis loops are illustrated.

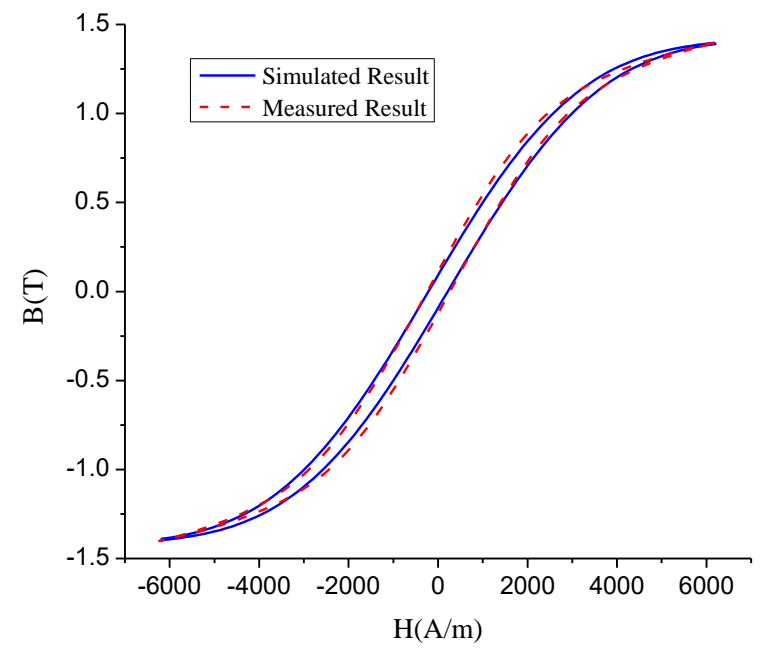

(a) 


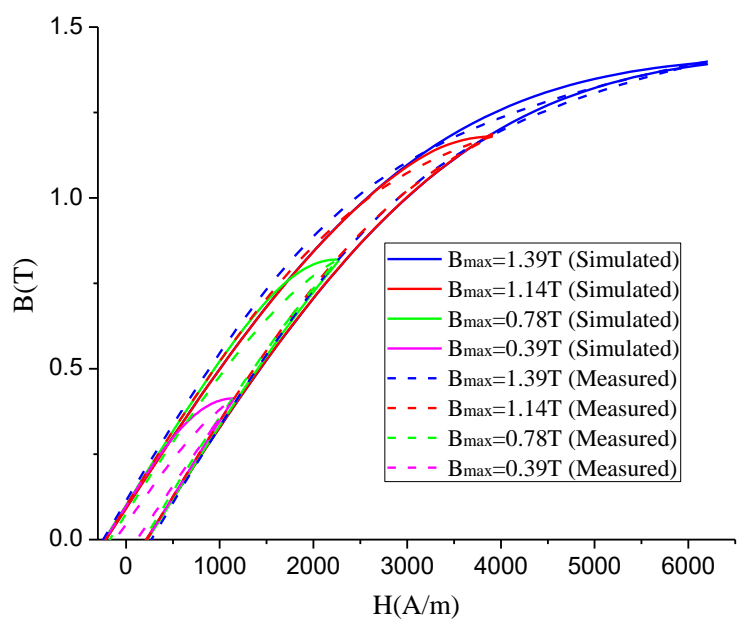

(b)

Fig. 16. Comparison between simulation and measurement of (a) limiting hysteresis loop and (b) major hysteresis loops with different flux densities under $50 \mathrm{~Hz}$.

Fig. 17 shows a series of $\mathbf{B}$ and $\mathbf{H}$ loci while the magnetic flux densities are controlled to form circular loci with magnitudes of $0.3,0.6,0.9,1.2,1.5 \mathrm{~T}$ at $50 \mathrm{~Hz}$, in which the measurement results are denoted with blue dashed lines and the simulation results with red solid lines respectively. The parameters in this case are obtained by fitting the model to the largest $\mathbf{B}$ and $\mathbf{H}$ loci. It is clear that the $\mathbf{B}$ loci are well controlled as the round loci, and the $\mathbf{H}$ loci change from circles to square-like loops with increasing magnitudes of $\mathbf{B}$.

The comparison demonstrates that although the simulated results do not match exactly with the simulated results, especially in the high flux density area, it is still acceptable and valid for developing high performance electromagnetic devices [32]. It is obvious that the simulations of the magnetic properties, especially the hysteresis, of SMC material under under different magnetic flux patterns agree in general with the measured results, which can prove the effectiveness of the proposed model.

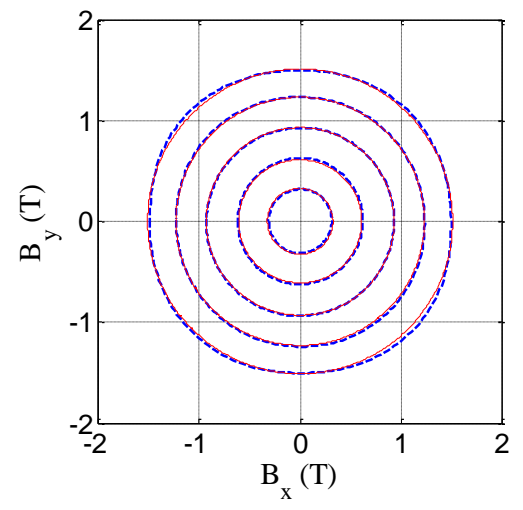

(a)

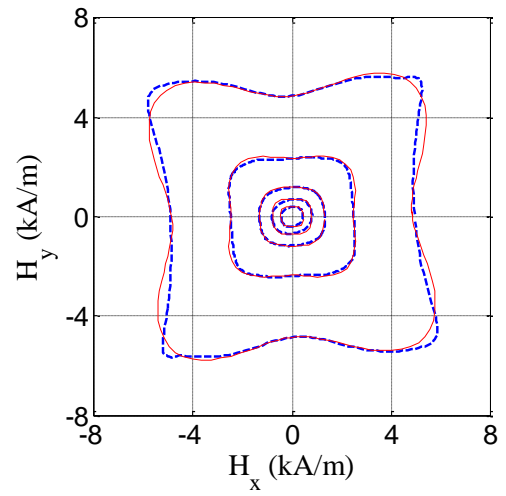

(b)

Fig. 17. The comparison of measurement results (blue dashed line) and simulation results (red solid line) about the loci of (a) $\mathbf{B}$ and (b) $\mathbf{H}$ in SOMALOY ${ }^{\mathrm{TM}} 500$ specimen with circular rotating flux density at $50 \mathrm{~Hz}$.

\section{CONCLUSION}

A vectorial elemental operator with biaxial anisotropy, based on the physical mechanisms of magnetic materials, was proposed to simulate the surface coated iron particles in the SMC material. Then, the magnetic properties of SMC material under different magnetic flux patterns can be determined by the superposition of these different elemental operators.

This paper presents also the measured magnetic property of a kind of SMC material under alternating and rotational fields, respectively, by using the 3-D magnetic property measurement system. The hysteresis phenomenon under rotational field is totally different from that under alternating field. The relationship of the vectorial $\mathbf{B}-\mathbf{H}$ cannot be described by a loop, but circular loci of $\mathbf{B}$ plus the corresponding flower-shaped loci of $\mathbf{H}$. With the help of the elemental operator and distribution function, both the magnitudes and directions of $\mathbf{B}$ and $\mathbf{H}$ can be described in this proposed model.

The results predicted by the proposed model agree well with the experimental results in SMC materials. This model is convenient for incorporation in the software packages for practical engineering electromagnetic field analysis, which can be helpful for design optimization of electromagnetic devices with SMC cores.

\section{REFERENCES}

[1] J. G. Zhu, Y. G. Guo, Z. W. Lin, et al., "Development of PM transverse flux motors with soft magnetic composite cores," IEEE Trans. Magn., vol. 47, DOI 10.1109/TMAG.2011.2157320, no. 10, pp. 4376-4383, Oct. 2011.

[2] Y. J. Li, Q. X. Yang, J. G. Zhu, and Y. G. Guo, "Magnetic properties measurement of soft magnetic composite materials over wide range of excitation frequency," IEEE Trans. Ind. Appl., vol. 48, DOI 10.1109/TIA.2011.2175677, no. 1, pp. 88-97, Nov. 2012.

[3] Y. G. Guo, J. G. Zhu, and D. G. Dorrell, "Design and analysis of a claw pole permanent magnet motor with molded soft magnetic composite core," IEEE Trans. Magn., vol. 45, DOI 10.1109/TMAG.2009.2022745 ,no. 10, pp. 4582-4585, Oct. 2009.

[4] G. Lei, T. S. Wang, J. G. Zhu, et al., "System level design optimization methods for electrical drive systems: robust approach," IEEE Trans. Ind. Electron, vol. 62, DOI 10.1109/TIE.2015.2404305, no. 8, pp.4702-4713, Aug. 2015.

[5] N. N. Duan, W. J. Xu, S. H. Wang, et al., "An improved XFEM with multiple high-order enrichment functions and low-order meshing elements for field analysis of electromagnetic devices with multiple 
nearby geometrical interfaces," IEEE Trans. Magn., vol. 51, DOI 10.1109/TMAG.2014.2358792, no. 3, art. 7206004, Mar. 2015.

[6] Y. J. Li, L, Cao, C. G. Zhang, et al., "Rotational core loss of silicon steel laminations based on three-dimensional magnetic properties measurement," IEEE Trans. Appl. Supercond., vol. 26, DOI 10.1109/TASC.2016.2532039, no. 4, art. 8201205, Jun. 2016.

[7] X. Sun, L. Chen, and Z. Yang, "Overview of bearingless permanent magnet synchronous motors," IEEE Trans. Ind. Electron., vol. 60, DOI 10.1109/TIE.2012.2232253, no. 12, pp. 5528-5538, Dec. 2013.

[8] X. Sun, L. Chen, and Z. Yang, H. Zhu, "Speed-sensorless vector control of a bearingless induction motor with artificial neural network inverse speed observer," IEEE/ASME Trans. Mechatronics, vol. 18, DOI 10.1109/TMECH.2012.2202123, no. 4, pp. 1357-1456, Aug. 2013.

[9] F. Marignetti, V. D. Colli, and Y. Coia, "Design of axial flux PM synchronous machines through 3-D coupled electromagnetic thermal and fluid-dynamical finite-element analysis," IEEE Trans. Ind. Electron, vol. 55, DOI 10.1109/TIE.2008.2005017, no. 10, pp. 3591-3601, Sep. 2008.

[10] G. Lei, T. S. Wang, Y. G. Guo, J. G. Zhu, and S. H. Wang, "System level design optimization methods for electrical drive systems: deterministic approach," IEEE Trans. Ind. Electron, vol. 61, DOI 10.1109/TIE.2014.2321338, no. 12, pp. 6591-6602, Dec. 2014.

[11] R. D. Stefano and F. Marignetti, "Electromagnetic analysis of axial-flux permanent magnet synchronous machines with fractional windings with experimental validation," IEEE Trans. Ind. Electron, vol. 59, DOI 10.1109/TIE.2011.2165458, no. 6, pp. 2573-2582, Aug. 2012.

[12] C. C. Liu, J. G. Zhu, Y. H. Wang, Y. G. Guo, and G. Lei, "Comparison of claw pole machines with different rotor structures," IEEE Trans. Magn., vol. 51, DOI 10.1109/TMAG.2015.2443022, no. 11, art. 8110904, Jun. 2015.

[13] Y. J. Li, Y. F. Li, et al., "Magnetic anisotropic properties measurement and analysis of the soft magnetic composite materials," IEEE Trans. Appl. Supercond., vol. 24, DOI 10.1109/TASC.2014.2348569, no. 5, pp. 1-4, Aug. 2014.

[14] C. C. Liu, J. G. Zhu, Y. H. Wang, et al., "Development of a low-cost double rotor axial flux motor with soft magnetic composite and ferrite permanent magnet materials," J. Appl. Phys., vol. 117, DOI 10.1063/1.4907235, no. 17, art. 17B507, May. 2015.

[15] J. G. Zhu and V. S. Ramsden, "Improved formulations for rotational core losses in rotating electrical machines," IEEE Trans. Magn., vol. 34, DOI 10.1109/20.703861, no. 4, pp. 2234-2242, Jul. 1998.

[16] Q. Yu, B. Bilgin, and A. Emadi, "Loss and efficiency analysis of switched reluctance machines using a new calculation method," IEEE Trans. Ind. Electron, vol. 62, DOI 10.1109/TIE.2015.2392716, no. 5, pp. 3072-3080, Jan. 2015.

[17] J. Pyrhönen, S. Ruoho, J. Nerg, et al., "Hysteresis losses in sintered NdFeB permanent magnets in rotating electrical machines," IEEE Trans. Ind. Electron, vol. 62, DOI 10.1109/TIE.2014.2354597, no. 2, pp. 857-865, Feb. 2015.

[18] Y. J. Li, Q. X. Yang, J. G. Zhu, et al., "Research of three-dimensional magnetic reluctivity tensor based on measurement of magnetic properties," IEEE Trans. Appl. Supercond., vol. 24, DOI 10.1109/TASC.2009.2039559, no. 3, pp. 1932-1935, Jun. 2010.

[19] E. C. Stoner and E. P. Wohlfarth, "A mechanism of magnetic hysteresis in heterogeneous alloys," IEEE Trans. Magn., vol. 27, DOI 10.1109/TMAG.1991.1183750, no.4, pp. 3475-3518, Jul.1991.

[20] N. N. Duan, W. J. Xu, S. H. Wang, et al., "Hysteresis modeling of high-temperature superconductor using simplified Preisach model," IEEE Trans. Magn., vol. 51, DOI 10.1109/TMAG.2014.2347962, no. 3, art.7300904, Mar. 2015.

[21] E. Cardelli, E. Della Torre, and A. Faba, "Analysis of a unit magnetic particle via the DPC model," IEEE Trans. Magn., vol. 45, DOI 10.1109/TMAG.2009.2031078, no. 11, pp. 5192-5195, Nov. 2009.

[22] N. N. Duan, W. J. Xu, S. H. Wang, et al., "A new Preisach type hysteresis model of high temperature superconductors," J. Appl. Phys., vol. 117, DOI 10.1063/1.4914124, no. 17, art. 17A718, 2015.

[23] G. R. Kahler, E. Della Torre and E. Cardelli, "Implementation of the Preisach-Stoner-Wohlfarth classical vector model," IEEE Trans. Magn., vol. 46, DOI 10.1109/TMAG.2009.2030676, no. 1, pp. 21-28, Jan. 2010.

[24] D. Li, F. Liu, Y. Li, Z. Zhao, C. Zhang, and Q. Yang, "Magnetic properties modeling of soft magnetic composite materials using two-dimensional vector hybrid hysteresis model," J. Appl. Phys., vol. 115, DOI 10.1063/1.4862841, no. 17, art. 17D117, 2014.

[25] D. C. Jiles, Introduction to Magnetism and Magnetic Materials. Florida, CRC press, 1998.
[26] G. Bertotti, Hysteresis in Magnetism: for Physicists, Materials Scientists, and Engineers. San Diego, Academic press, 1998.

[27] I. Petrila and A. Stancu, "Hysteresis characteristics of an analytical vector hysteron," Physica. B, vol. 406, DOI 10.1016/j.physb.2010.12.025, no. 4, pp. 906-910, Feb. 2011

[28] Y. G. Guo, J. G. Zhu, Z. W. Lin, et al., "3D vector magnetic properties of soft magnetic composite material," J. Magn. Magn. Mater., vol. 302, DOI 10.1016/j.jmmm.2005.10.019, no. 2, pp. 511-516, Jul. 2006.

[29] Y. J. Li, Q. X. Yang, J. G. Zhu, et al., "Measurement of soft magnetic composite material using an improved 3-D tester with flexible excitation coils and novel sensing coils," IEEE Trans. Magn., vol. 46, DOI 10.1109/TMAG.2010.2043339, no. 6, pp. 1971-1974, Jun. 2010.

[30] Y. J. Li, Q. X. Yang, J. G. Zhu, et al., "Design and analysis of a novel 3-D magnetization structure for laminated silicon steel,". IEEE Trans. Magn., vol. 50, DOI 10.1109/TMAG.2013.2284097, no. 2, art. 7009504, Feb. 2014.

[31] Y. J. Li, J. G. Zhu, Q. X. Yang, , et al., "Study on rotational hysteresis and core loss under three dimensional magnetization," IEEE Trans. Magn., vol. 47, DOI 10.1109/TMAG.2011.2153186, no. 10, pp. 3520-3523, Oct. 2011

[32] Y. G. Guo, J. G. Zhu, J. J. Zhong, et al., "Measurement and modeling of rotational core losses of soft magnetic materials used in electrical machines: A review," IEEE Trans. Magn. , vol. 44, DOI 10.1109/TMAG.2007.911250, no. 2, pp. 279-291, Feb. 2008.

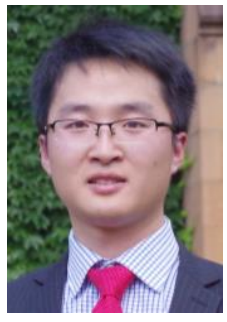

Weijie Xu (S'15) received the B.E. degree from Henan Polytechnic University, Jiaozuo, China, in 2009. From December 2013 to November 2015, he was a visiting $\mathrm{Ph} . \mathrm{D}$. student in the University of Technology Sydney (UTS).

$\mathrm{He}$ is currently working toward the Ph.D. degree at Xi'an Jiaotong University. His research interests include measurement and modeling of properties of novel magnetic materials, and new material applications in electromagnetic devices.

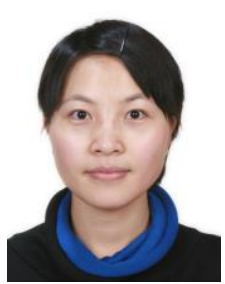

Nana Duan (S'15) received the B.E. degree from Henan Polytechnic University, Jiaozuo, China, in 2009, and the $\mathrm{Ph} . \mathrm{D}$. degree in electrical engineering from Xi'an Jiaotong University, Xi'an, China, in 2016. From January 2013 to January 2015, she was a visiting Ph.D. student in the University of Technology Sydney (UTS).

She is currently a Lecturer with the School of Electrical Engineering, Xi'an Jiaotong University. Her research fields include numerical analysis of electromagnetic field and the electromagnetic analysis for high temperature superconducting power cable.

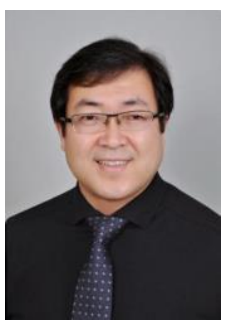

Shuhong Wang (M'11-SM'13) received the B.E., M.E., and $\mathrm{Ph} . \mathrm{D}$. degrees in electrical engineering from $\mathrm{Xi}^{\prime}$ 'an Jiaotong University, Xi'an, China, in 1990, 1993, and 2002, respectively. 
He is currently a Professor with the school of Electrical Engineering, Xi'an Jiaotong University. His research fields include numerical analysis of electromagnetic field, design and optimization of electromagnetic devices, measurement and modeling of properties of novel magnetic materials and the electromagnetic analysis for high temperature superconducting power cable.

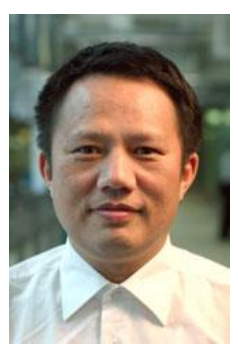

Youguang Guo (S'02-M'05-SM'06) received the B.E. degree from Huazhong University of Science and Technology, Wuhan, China, in 1985, the M.E. degree from Zhejiang University, Hangzhou, China, in 1988, and the Ph.D. degree from the University of Technology Sydney (UTS), Sydney, Australia, in 2004, all in electrical engineering.

$\mathrm{He}$ is currently an Associate Professor with the School of Electrical, Mechanical and Mechatronic Systems, UTS. His research fields include measurement and modeling of properties of magnetic materials, numerical analysis of electromagnetic field, electrical machine design optimization, power electronic drives, and motor control.

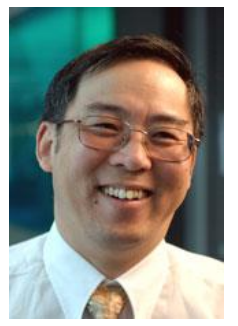

Jianguo Zhu (S'93-M'96-SM'03) received the B.E. degree from Jiangsu Institute of Technology, Zhenjiang, China, in 1982, the M.E. degree from Shanghai University of Technology, Shanghai, China, in 1987, and the $\mathrm{Ph} . \mathrm{D}$. degree from the University of Technology Sydney (UTS), Sydney, Australia, in 1995, all in electrical engineering.

$\mathrm{He}$ is currently a Professor of electrical engineering and the Head of the School of Electrical, Mechanical and Mechatronic Systems with the UTS. His current research interests include electromagnetics, magnetic properties of materials, electrical machines and drives, power electronics, and green energy systems. 